$\mathrm{e}^{+} \mathrm{e}^{-}$Collisions from Phi to Psi 2013 (PHIPSI2013)

International Journal of Modern Physics: Conference Series

Vol. 35 (2014) 1460442 (6 pages)

(C) The Author

DOI: $10.1142 /$ S2010194514604426

\title{
PERTURBATIVE EXPANSION OF $\tau$ HADRONIC SPECTRAL FUNCTION MOMENTS
}

\author{
DIOGO BOITO \\ Physik Department T31, Technische Universität München \\ James-Frack-Straße 1, D-85748 Garching, Germany
}

Published 18 December 2014

\begin{abstract}
In the extraction of $\alpha_{s}$ from hadronic $\tau$ decay data several moments of the spectral functions have been employed. Furthermore, different renormalization group improvement (RGI) frameworks have been advocated, leading to conflicting values of $\alpha_{s}$. Recently, we performed a systematic study of the perturbative behavior of these moments in the context of the two main-stream RGI frameworks: Fixed Order Perturbation Theory (FOPT) and Contour Improved Perturbation Theory (CIPT). The yet unknown higher order coefficients of the perturbative series were modelled using the available knowledge of the renormalon singularities of the QCD Adler function. We were able to show that within these RGI frameworks some of the commonly employed moments should be avoided due to their poor perturbative behavior. Furthermore, under reasonable assumptions about the higher order behavior of the perturbative series FOPT provides the preferred RGI framework.
\end{abstract}

Keywords: $\tau$-decays; renormalization group; renormalons.

PACS Numbers: 13.35.Dx, 12.38.Cy

\section{Introduction, framework, and results}

The determination of $\alpha_{s}$ from hadronic $\tau$ decays is one of the most precise determinations of the QCD coupling. ${ }^{1,2}$ In the inclusive $\tau$ hadronic width, $R_{\tau}$, in spite of the relatively low energy scale set by the $\tau$ mass, the non-perturbative contribution is subleading and the theoretical description is dominated by perturbative QCD. In detailed $\alpha_{s}$ analyses, one exploits the knowledge of the spectral functions, measured by OPAL and ALEPH at LEP, ${ }^{3,4}$ in order to construct additional observables. Different moments of the spectral functions are used; their theoretical counterpart is evaluated through finite energy sum-rules, as contour integrals in the complex-energy plane. In this context, $R_{\tau}$ can be understood as a particular choice of moment of the spectral functions integrated up to the kinematical limit $s_{0}=m_{\tau}^{2}$.

This is an Open Access article published by World Scientific Publishing Company. It is distributed under the terms of the Creative Commons Attribution 3.0 (CC-BY) License. Further distribution of this work is permitted, provided the original work is properly cited. 
Other analytic weight functions and upper limits $s_{0} \leq m_{\tau}^{2}$ (as long as $s_{0}$ is large enough to allow a perturbative treatment) also define observables that can be computed theoretically. The use of tailored weight functions can be instrumental to the $\alpha_{s}$ analysis, e.g., suppressing or enhancing the non-perturbative contributions. ${ }^{3-8}$

Our focus is on the perturbative QCD contribution to the different moments used in $\alpha_{s}$ analyses. Recently, ${ }^{9,10}$ we investigated the convergence of the perturbative series after integration in the complex plane employing two different renormalization group improvement (RGI) prescriptions and discussed how the convergence properties of the series depend on the specific moment used. Here we briefly describe the methods and the results of our analysis and try to summarize the main conclusions.

For the theoretical description, the relevant quantity is the QCD Adler function, which is renormalization group ( $\mathrm{RG}$ ) invariant. The perturbative contribution to the observable defined by the weight function $w_{i}(s)$, denoted $\delta_{w_{i}}^{(0)}$, is obtained through an integration on the complex energy plane along the circle of radius $s_{0}$. Defining $x=s / s_{0}, W_{i}(x)=2 \int_{x}^{1} w_{i}(z) d z$, and $a_{\mu} \equiv a\left(\mu^{2}\right) \equiv \alpha_{s}(\mu) / \pi$, the explicit expression reads

$$
\delta_{w_{i}}^{(0)}=\sum_{n=1}^{\infty} \sum_{k=1}^{n} k c_{n, k} \frac{1}{2 \pi i} \oint_{|x|=1} \frac{d x}{x} W_{i}(x) \log ^{k-1}\left(\frac{-s_{0} x}{\mu^{2}}\right) a_{\mu}^{n} .
$$

The dynamical input to this series is fully contained in the $c_{n, 1}$ coefficients, known at present up to $\alpha_{s}^{4}$ order. ${ }^{11}$ The other coefficients can be determined using RG invariance in terms of the $c_{n, 1}$ and $\beta$-function coefficients.

The scale $\mu$ in the last equation can be set in a convenient way due to RG invariance. The two mainstream choices are known as fixed-order perturbation theory ${ }^{12}$ (FOPT) obtained by fixing the scale $\mu^{2}=s_{0}$, and contour improved perturbation theory $^{13,14}$ (CIPT) obtained when the running of $\alpha_{s}$ is resummed along the contour by setting $\mu^{2}=x s_{0}$. (The FOPT series can be reobtained from CIPT via the expansion of the running coupling $a\left(x s_{0}\right)$ in terms of the coupling at a fixed scale $\mu^{2}=s_{0}$.) Both expansions are expected to diverge at large orders due to factorial growth of the perturbative coefficients. Therefore, the two series define two different asymptotic expansions (at best) to the value of the $\delta_{w_{i}}^{(0)}$. In practice, the numerical differences are large at $\alpha_{s}^{4}$ which represents one of the dominant sources of theoretical uncertainty.

A comparison between the two approaches regarding their success in approximating $\delta_{w_{i}}^{(0)}$ depends on assumptions about the higher order terms. A strategy to deal with this problem based on the available knowledge of the renormalon singularities of the Borel transformed Adler function was put forward by Beneke and Jamin. ${ }^{15}$ They were able to show that under reasonable assumptions - to be discussed below - FOPT is to be preferred for the inclusive $\tau$ hadronic width. Later we extended this analysis 9,10 in order to ascertain how the behavior of the perturbative series depends on the moment $w_{i}(x)$ as well as on the value of $s_{0}$. 
We work with the Adler function $\widehat{D}$, which contains only the corrections to the parton model result, and define its Borel transform $B[\widehat{D}](t)$ as

$$
\widehat{D}(s) \equiv \sum_{n=0}^{\infty} r_{n} \alpha_{s}(\sqrt{s})^{n+1}, \quad \text { and } \quad B[\widehat{D}](t) \equiv \sum_{n=0}^{\infty} r_{n} \frac{t^{n}}{n !},
$$

with $c_{n, 1}=\pi^{n} r_{n-1}$. The original series $\widehat{D}$ can be understood as an asymptotic expansion of the inverse of $B[\widehat{D}](t)$

$$
\widehat{D}(\alpha) \equiv \int_{0}^{\infty} e^{-t / \alpha} B[\widehat{D}](t),
$$

when the integral exists. This equation defines the Borel sum of the series.

The Borel transformed Adler function has singularities along the real axis both for negative and positive values of $t$, known as renormalon singularities (for a review see $^{16}$ ). General RG arguments and the structure of the OPE allows one to determine the position and, in principle, the strength of these singularities - the residues are unknown. Singularities on the positive real axis, infrared (IR) renormalons, give rise to fixed sign divergent series. These singularities obstruct the integration in Eq. (3) and produce an ambiguity in the Borel sum related to the prescription used to define the integral. Singularities for $t<0$, ultraviolet (UV) renormalons, give rise to sign alternating divergent series. The fixed sign nature of the exactly known coefficients of the Adler function suggests that the series is dominated by IR singularities at low and intermediate orders.

The strategy then consists in constructing a model for the Borel transformed Adler function containing a small number of dominant renormalon singularities whose residues are unknown. The residues are then fixed in order to reproduce the known coefficients and an estimate of the $\alpha_{s}^{5}$ term. The Adler function can be reconstructed to all orders and the RG improved result can be compared with a "true" result for $\delta_{w_{i}}^{(0)}$, obtained using Eq. (3). The main assumptions behind this strategy is that the series exhibits some regularity, and that sufficiently many terms are known in order to fix the contribution of the leading renormalons. This has been tested in detail using the large- $\beta_{0}$ limit of QCD and the plausibility of these assumptions has been confirmed. ${ }^{9}$

Two models were constructed. ${ }^{9,15}$ The first, and more realistic one in our opinion, assumes a logical hierarchy between the IR renormalon contributions. It includes the two leading IR renormalons along with the leading UV (whose signature is not yet seen in the known coefficients). This gives rise to a model that we dub the reference model (RM). This model is contrasted with a second where the leading IR renormalon is artificially removed from the Borel transformed, which realizes the case where the contributions of the second IR renormalon are maximized. This is called the alternative model (AM). Using these two models for the higher orders, we performed a systematic analysis of a collection of different moments, using different $s_{0}$ values, and comparing the performance of FOPT and CIPT. As an example, Figs. 1 and 2 show results for the kinematic moment within the two models. ${ }^{9,10}$ 


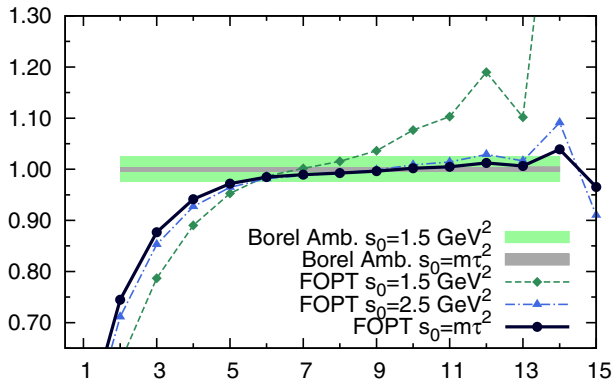

(a) $w_{\tau}(x)=(1-x)^{2}(1+2 x)$, FOPT.

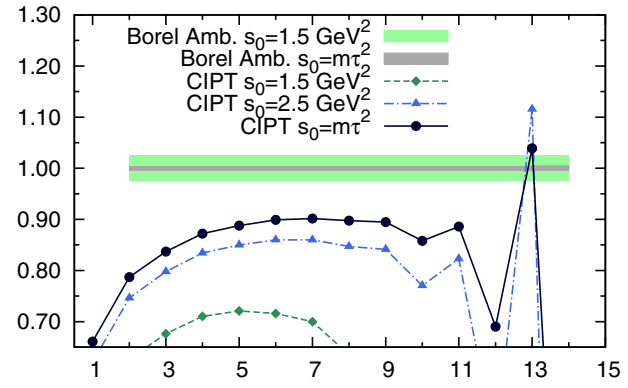

(b) $w_{\tau}(x)=(1-x)^{2}(1+2 x)$, CIPT.

Fig. 1. Reference model. $\delta_{w_{\tau}}^{(0)}\left(s_{0}\right)$ order by order in $\alpha_{s}$ normalised to the Borel sum for FOPT (left) and CIPT (right) with three values of $s_{0}: 1.5 \mathrm{GeV}^{2}, 2.5 \mathrm{GeV}^{2}$, and $m_{\tau}^{2}$. Bands give the Borel ambiguities.

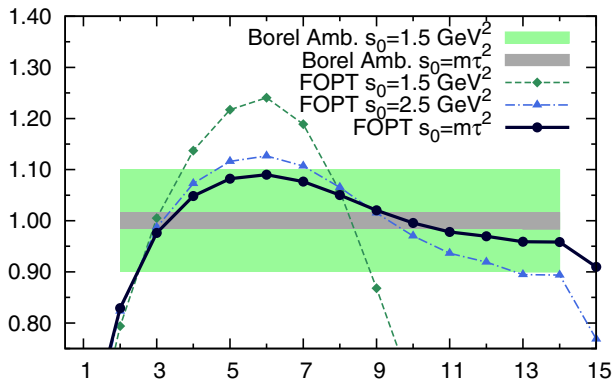

(a) $w_{\tau}(x)=(1-x)^{2}(1+2 x)$, FOPT.

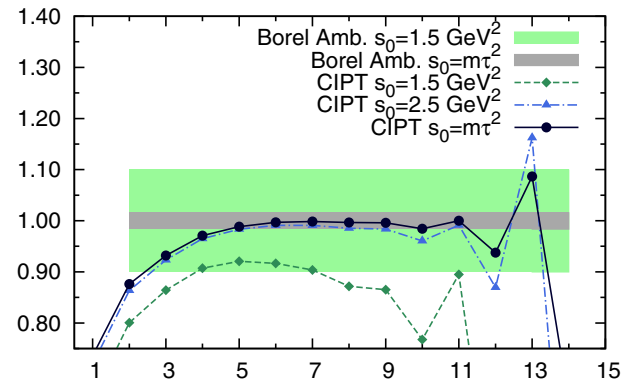

(b) $w_{\tau}(x)=(1-x)^{2}(1+2 x)$, CIPT.

Fig. 2. Alternative model. $\delta_{w_{\tau}}^{(0)}\left(s_{0}\right)$ order by order in $\alpha_{s}$ normalised to the Borel sum for FOPT (left) and CIPT (right) with three values of $s_{0}: 1.5 \mathrm{GeV}^{2}, 2.5 \mathrm{GeV}^{2}$, and $m_{\tau}^{2}$. Bands give the Borel ambiguities.

They clearly show the preference for FOPT within the more realistic RM. CIPT gives the better approximation when the leading IR renormalon is artificially suppressed.

\section{Conclusions}

Here we try to summarize our main conclusions. ${ }^{9,10,15}$

- The finiteness of the radius of convergence of the expansion of the running coupling $^{14}$ in terms of $\alpha_{s}\left(\sqrt{s_{0}}\right)$ does not disfavor FOPT, for the perturbative series of $\widehat{D}$ is expected to be divergent. The models corroborate this conclusion: FOPT provides a better asymptotic expansion than CIPT in the case of the RM.

- CIPT and FOPT define two different asymptotic series. FOPT treats the running of $\alpha_{s}$ and the $c_{n, 1}$ coefficients on an equal footing and only includes at a 
given order $n$ terms up to order $\alpha_{s}\left(\sqrt{s_{0}}\right)^{n}$. In CIPT the running of $\alpha_{s}$ is always resummed to all orders although only a finite number of $c_{n, 1}$ coefficients contribute at a given order. Contrary to what is often stated, there is no reason to believe that the differences in the $\alpha_{s}$ values from FOPT and CIPT can be attributed to missing higher orders.

- The preference for FOPT or CIPT can be mapped into an assumption about the renormalon content of the QCD Adler function. FOPT is superior whenever a sizable contribution from the leading $(D=4)$ IR renormalon is present. The naturalness of this scenario is a strong argument in favor of FOPT. The (artificial) suppression of this contribution realizes a scenario where CIPT is superior.

- In the context of the RGI frameworks discussed here, some of the moments that are commonly employed in determinations of $\alpha_{s}$ should be avoided due to their poor perturbative behavior. In particular, the moments that emphasise higher OPE condensates $(D \geq 8)$ used in $^{3-5}$ should be avoided. In contrast, the moments used in ${ }^{6-8}$ have a better convergence, and at least one of the series (FOPT or CIPT) approaches the "true" value at relatively low orders.

- Ideally, the goodness of the RGI framework should be moment independent. Preferentially, it should also be independent on the assumptions about the renormalon singularities of the Adler function (in the context of our work this can be rephrased as being model independent). To our knowledge, the most promising strategy in this direction is the use of conformal mapping techniques based on the partial knowledge of the Borel transformed Adler function. ${ }^{17}$

\section{Acknowledgements}

It is a pleasure to thank Martin Beneke and Matthias Jamin for the collaboration in this subject and for the reading of the manuscript. This work was supported by the Alexander von Humboldt Foundation.

\section{References}

1. E. Braaten, S. Narison, and A. Pich, Nucl. Phys. B 373 (1992) 581.

2. M. Davier, A. Höcker and Z. Zhang, Rev. Mod. Phys. 78 (2006) 1043.

3. K. Ackerstaff et al. [OPAL Collaboration], Eur. Phys. J. C 7 (1999) 571.

4. R. Barate et al. [ALEPH Collaboration], Eur. Phys. J. C 4, 409 (1998).

5. M. Davier, S. Descotes-Genon, A. Höcker, B. Malaescu, and Z. Zhang, Eur. Phys. J. C 56 (2008) 305; M. Davier, A. Hoecker, B. Malaescu, C. Yuan and Z. Zhang, arXiv:1312.1501 [hep-ex].

6. K. Maltman and T. Yavin, Phys. Rev. D 78 (2008) 094020.

7. D. Boito, O. Catà, M. Golterman, M. Jamin, K. Maltman, J. Osborne, and S. Peris, Phys. Rev. D 84 (2011) 113006.

8. D. Boito, M. Golterman, M. Jamin, A. Mahdavi, K. Maltman, J. Osborne, and S. Peris, Phys. Rev. D 85 (2012) 093015.

9. M. Beneke, D. Boito and M. Jamin, JHEP 1301 (2013) 125.

10. D. Boito, PoS ConfinementX , 044 (2012) [arXiv:1301.3008 [hep-ph]].

11. P. A. Baikov, K. G. Chetyrkin and J. H. Kühn, Phys. Rev. Lett. 101 (2008) 012002. 
12. see, e.g., M. Jamin, JHEP 0509 (2005) 058.

13. A. A. Pivovarov, Z. Phys. C 53 (1992) 461 [Sov. J. Nucl. Phys. 54 (1991) 676] [Yad. Fiz. 54 (1991) 1114].

14. F. Le Diberder and A. Pich, Phys. Lett. B 289 (1992) 165.

15. M. Beneke and M. Jamin, JHEP 0809 (2008) 044.

16. M. Beneke, Phys. Rept. 317 (1999) 1.

17. G. Abbas, B. Ananthanarayan, I. Caprini and J. Fischer, Phys. Rev. D 88 (2013) 034026 . 